mary and secondary iron overload disorders: preclinical models and approaches. Hematol Oncol Clin North Am. 2014;28(2):387-401.

15. Preza GC, Ruchala P, Pinon R, et al. Minihepcidins are rationally designed small peptides that mimic hepcidin activity in mice and may be useful for the treatment of iron overload. J Clin Invest. 2011;121(12):4880-4888.

16. Ramos E, Ruchala P, Goodnough JB, et al. Minihepcidins prevent iron overload in a hepcidin-deficient mouse model of severe hemochromatosis. Blood. 2012;120(18):3829-3836.

17. Casu C, Oikonomidou PR, Chen H, et al. Minihepcidin peptides as disease modifiers in mice affected by beta-thalassemia and polycythemia vera. Blood. 2016;128(2):265-276.

18. Casu C, Chessa R, Liu A, et al. Minihepcidins improve ineffective erythropoiesis and splenomegaly in a new mouse model of adult $\beta$ thalassemia major. Haematologica. 2020;105(7):1835-1844.

19. Camaschella C. Treating iron overload. N Engl J Med. 2013;368(24): 2325-2327.

20. Coates TD. Iron overload in transfusion-dependent patients.
Hematology Am Soc Hematol Educ Program. 2019;2019(1):337-344 21. Angelucci E. Hematopoietic stem cell transplantation in thalassemia. Hematology Am Soc Hematol Educ Program. 2010;2010:456-462.

22. Thompson AA, Walters MC, Kwiatkowski J, et al. Gene therapy in patients with transfusion-dependent beta-thalassemia. N Engl J Med. 2018;378(16):1479-1493.

23. Galanello R, Origa R. Beta-thalassemia. Orphanet J Rare Dis. 2010;5:11.

24. Casu C, Nemeth E, Rivella S. Hepcidin agonists as therapeutic tools. Blood. 2018;131(16):1790-1794.

25. Camaschella C, Nai A. Ineffective erythropoiesis and regulation of iron status in iron loading anaemias. Br J Haematol. 2016;172(4):512-523.

26. Cappellini MD, Viprakasit V, Taher AT, et al. A phase 3 trial of luspatercept in patients with transfusion-dependent beta-thalassemia. N Engl J Med. 2020;382(13):1219-1231.

27. Busti F, Marchi G, Lira Zidanes A, Castagna A, Girelli D. Treatment options for anemia in the elderly. Transfus Apher Sci. 2019;58(4):416-421.

\title{
ABL-class fusion positive acute lymphoblastic leukemia: can targeting ABL cure ALL?
}

\section{Thai Hoa Tran ${ }^{1}$ and Stephen P. Hunger ${ }^{2}$}

${ }^{1}$ Division of Pediatric Hematology-Oncology, Charles-Bruneau Cancer Center, CHU Sainte-Justine, University of Montreal, Montreal, Quebec, Canada and 'Department of Pediatrics, The Center for Childhood Cancer Research, Children's Hospital of Philadelphia, The Perelman School of Medicine, University of Pennsylvania, Philadelphia, PA, USA

E-mail: STEPHEN HUNGER - hungers@email.chop.edu

doi:10.3324/haematol.2020.252916

$\mathrm{F}$ ive-year survival rates for pediatric acute lymphoblastic leukemia (ALL), a malignancy that was incurable in the 1950s, now exceed $90 \% .{ }^{1}$ However, $15-20 \%$ of National Cancer Institute (NCI) high-risk (HR) B-lineage ALL (B-ALL) patients relapse, and post-relapse outcomes remain poor, particularly following early marrow relapse [5-year overall survival (OS): 28\%]. ${ }^{2}$ Genomic advances have identified a novel B-ALL subtype characterized by a heterogeneous spectrum of kinase-activating alterations, producing a gene expression signature similar to that of Philadelphia chromosome-positive $\left(\mathrm{Ph}^{+}\right) \mathrm{ALL}$, without the canonical BCR-ABL1 oncoprotein, referred to as BCR-ABL1-like ALL or Ph-like ALL, and now recognized as a provisional disease entity in the 2016 World Health Organization's classification of acute leukemias., Ph-like ALL is associated with adverse clinical features and poor outcomes despite modern therapy. ${ }^{4-6}$ It occurs in approximately $15 \%$ of children with NCI HR B-ALL and over $25 \%$ of adults with B-ALL, and contributes disproportionately to relapses. ${ }^{4,6}$ Among Ph-like ALL patients, $10-14 \%$ of them harbor rearrangements of ABL-class genes (ABL1, ABL2, CSF1R, LYN, PDGFRA, PDGFRB) other than $B C R-A B L 1$, collectively representing $2-3 \%$ of pediatric B-ALL cases. ${ }^{4-6}$ While there are anecdotal reports of the short-term efficacy of adding the ABL tyrosine kinase inhibitors (TKI) imatinib or dasatinib to chemotherapy, ${ }^{4,7,8}$ controlled data are lacking regarding the long-term efficacy of this approach. In this issue of Haematologica, Cario et al. ${ }^{9}$ provide important new information on treatment of children with ALL and ABL-class fusions.

They report $46 \mathrm{ABL}$-class fusion positive $\mathrm{B}$-ALL patients (15 involving ABL1, 5 ABL2, 3 CSF1R, and 23
PDGFRB) who were originally enrolled on the AIEOPBFM ALL 2000 and 2009 trials, and identified retrospectively. ABL-class fusion-positive cases had a substantially worse early treatment response than other patients, as reflected by prednisone-poor response $(50 \%$ vs. $5.6 \%$, $P<0.0001)$ or minimal residual disease $(\mathrm{MRD}) \geq 5 \times 10^{-4}$ at end-induction $(71.4 \%$ vs. $19.2 \%, P<0.0001)$ and end-protocol Ib $(51.2 \%$ vs. $5.1 \%, P<0.0001)$. Thirty-six of 46 patients $(78.3 \%)$ were classified as HR (vs. $11.1 \%$ of ALLBFM 2000 B-ALL patients overall), and more than half (25 of $46,54.3 \%$ ) underwent hematopoietic stem cell transplantation (HSCT) in first complete remission (CR1). For the cohort of 46 patients with ABL-class fusions, the 5year event-free survival (EFS) and OS were $49.1 \pm 8.9 \%$ and $69.6 \pm 7.8 \%$, respectively. The 5 -year cumulative incidence of relapse (CIR) and treatment-related mortality (TRM) were $25.6 \pm 8.2 \%$ and $20.8 \pm 6.8 \%$, respectively. Thirteen patients (13 of $46,28.3 \%$ ) received TKI in combination with chemotherapy post-induction; their outcomes were not significantly different from those in the no-TKI group ( $\mathrm{n}=33$ ) (5-year EFS $62.9 \%$ vs. $47.7 \%$, $P=0.98$; 5 -year OS $75.5 \%$ vs. $70.9 \%, P=0.64$ ). In parallel, ABL-class patients treated with or without HSCT had similar outcomes (5-year EFS $47.9 \%$ vs. $55.0 \%, P=0.35 ; 5$ year OS $66.7 \%$ vs. $84.0 \%, P=0.22)$. Notably, in the 33 patients treated without TKI, there was a trend towards lower CIR rate among patients who underwent HSCT $(n=16)$ compared to those who did not $(n=17)(13.2 \%$ vs. $43.8 \%, P=0.06$ ). The TRM rate was, nevertheless, exceedingly high in the HSCT group $(32.3 \%$ vs. $0.0 \%$, $P=0.034)$. Furthermore, the majority of events in the HSCT group were non-relapse events, while relapses predominate in the no-HSCT group. 
This article is valuable to clinicians as it confirms the adverse presenting features, poor early response and $\mathrm{EFS} / \mathrm{OS}$ rates of a large cohort of ABL-class fusion positive B-ALL patients, similar to prior anecdotal reports ${ }^{7}$ or smaller retrospective series. ${ }^{8,10}$ Moreover, the authors highlight the striking clinical resemblance between ABLclass fusion positive B-ALL and $\mathrm{Ph}^{+} \mathrm{ALL}$. Both disease entities comprise approximately $3 \%$ of pediatric ALL, tend to be older patients with hyperleukocytosis, elevat- ed MRD at the end of induction and consolidation, and the reported 5 -year EFS of less than $50 \%$ for the ABLclass cohort mirrors that of $\mathrm{Ph}^{+} \mathrm{ALL}$ in the pre-TKI era treated with the same chemotherapy backbone. ${ }^{11,12}$ This has important therapeutic implications, suggesting that the addition of TKI to chemotherapy, which has transformed survival of $\mathrm{Ph}^{+} \mathrm{ALL}$, may similarly translate into improved outcomes for ABL-class patients. ${ }^{13,14}$ In this series, six of the eight ABL-class patients who started TKI

\begin{tabular}{|c|c|c|c|c|c|}
\hline \multicolumn{2}{|c|}{ A } & $\mathrm{NCl} \mathrm{SR}{ }^{21}$ & $\begin{array}{c}\text { Childhood } \\
\mathrm{NCl} \mathrm{HR}\end{array}$ & $1-39$ yo 4 & $21-86$ yo $^{6}$ \\
\hline & $\mathrm{N}=$ total \# of B-ALL & 1023 & 1389 & 1725 & 798 \\
\hline & Ph-like ALL (\%) & $139(13.6)$ & $284(20.4)$ & $264(15.3)$ & $194(24.3)$ \\
\hline & $\mathrm{Ph}^{+} \mathrm{ALL}(\%)$ & $6(0.6)$ & $46(3.3)$ & $109(6.3)$ & $185(23.2)$ \\
\hline & ABL-class fusions (\%) & $2(0.2)$ & $40(2.9)$ & $36(2.0)$ & $19(2.4)$ \\
\hline
\end{tabular}

B

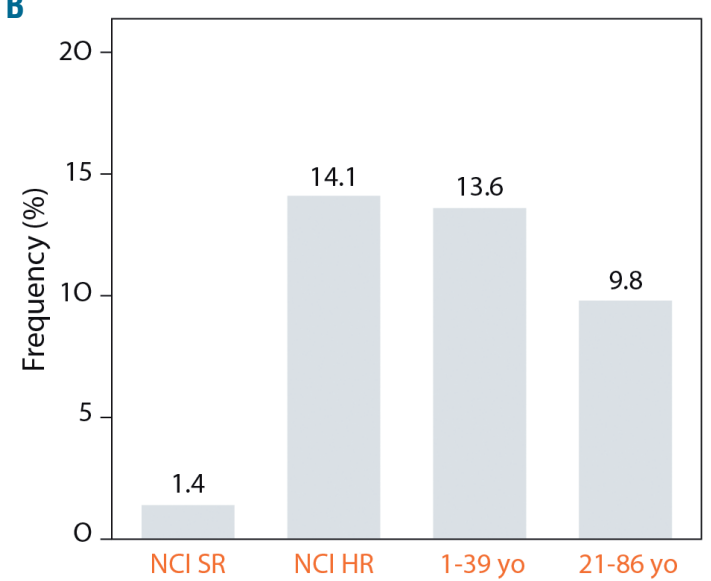

D

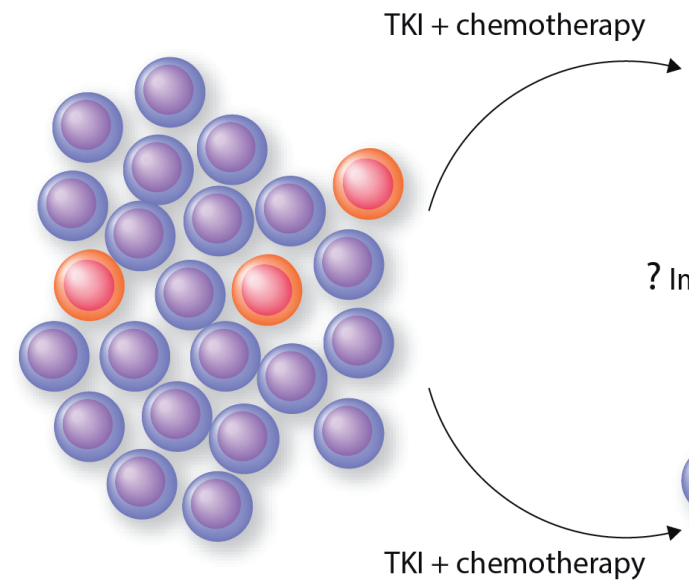

C

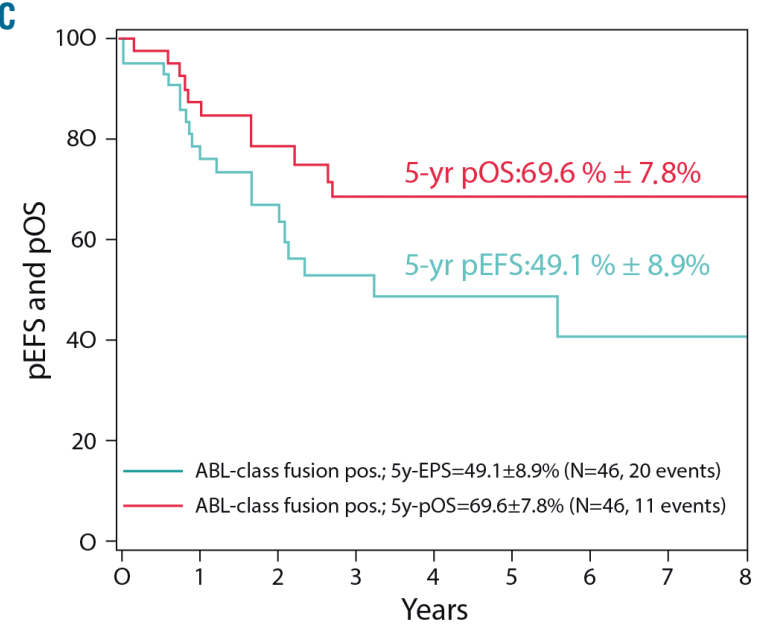

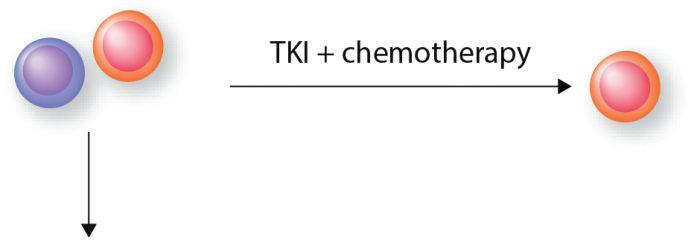

Immunotherapy +/-TKI

Figure 1. (A) Frequency of Philadelphia chromosome (Ph)-positive acute lymphoblastic leukemia (ALL), Ph-like ALL and ABL-class fusions in B-lineage ALL (B-ALL) according to National Cancer Institute $(\mathrm{NCl})$ risk status and age group, based on the following studies: Roberts et al., ${ }^{21}$ Reshmi et al., ${ }^{5}$ Roberts et al., ${ }^{4}$ and Roberts et al. ${ }^{6} \mathrm{NCl}$ SR: National Cancer Institute Standard Risk; NCl HR: National Cancer Institute High Risk. (B) Frequency of ABL-class fusions in Ph-like ALL. (C) Outcomes of ABL-class fusion positive B-ALL patients treated on the AIEOP-BFM ALL 2000 and 2009 trials. pEFS: projected event-free survival; pOS: projected overall survival. (D) Proposed treatment paradigm for ABL-class fusion positive B-ALL. Early introduction of tyrosine kinase inhibitor (TKI) to induction chemotherapy to achieve remission. Good-responders may continue with TKI and post-induction chemotherapy. Poor-responders will undergo allogeneic hematopoietic stem cell transplantation in first remission (CR1). Incorporation of immunotherapy with or without TKI is being considered in future trials to improve outcomes. yr: year. 
during consolidation had a low positive or negative endIb MRD, emphasizing the importance of early identification of ABL-class fusions and prompt TKI addition to achieve deep MRD response and, potentially, better outcomes. Despite the heterogeneity of ABL-class fusions, Cario et al. demonstrated that most ABL-class rearrangements could be detected by techniques such as fluorescence in situ hybridization (FISH) or polymerase chain reaction (PCR), which are standard techniques in clinical laboratories. Much effort in recent years has focused on screening for the kinase-activated signature that defines Ph-like ALL; however, ultimately, the clinically relevant goal is the prompt detection of the underlying therapeutically targetable genomic lesions. The Children's Oncology Group (COG) is now expanding their FISH panel to include $A B L 1, A B L 2$ and PDGFRB/CSF1R dualcolored break-apart probes to screen for ABL-class gene rearrangements in order to introduce TKI by mid-induction. This strategy could perhaps overcome the high rates of induction failure and eradicate MRD levels early in the course of therapy for the majority of ABL-class patients, as early TKI introduction has done for $\mathrm{Ph}^{+}$ALL. ${ }^{14,15}$ Prospective evaluation of the early addition of TKI to therapy of patients with ABL-class lesions is required; this can only be achieved by harnessing international collaborations to effectively design precision medicine trials for such rare disease entities as exemplified by the $\mathrm{Ph}^{+} \mathrm{ALL}$ experience (clinicaltrials.gov identifers: NCT0146016 and NCT03007147).

The article by Cario et al. also raises two fundamental questions which underlie the role of HSCT and the optimal chemotherapy backbone for ABL-class fusion positive B-ALL. HSCT appears to be an effective modality for disease control as fewer relapses occurred among ABLclass patients in the no-TKI group who underwent HSCT in CR1 (13.2\% vs. $43.8 \%, P=0.06)$. A single-center study previously reported comparable outcomes between children with Ph-like ALL and non-Ph-like ALL (5-year EFS $90.0 \%$ vs. $88.4 \%, P=0.41$, respectively), using $\mathrm{MRD}$ directed therapy intensification for relevant patients. ${ }^{16}$ Consequently, a significant higher proportion of Ph-like ALL patients underwent HSCT in CR1 due to end-induction MRD levels $\geq 1 \% \cdot{ }^{16}$ Nevertheless, HSCT is associated with unacceptably high TRM rates, which account for a considerable proportion of events in this AIEOP-BFM retrospective cohort. Given that ABL-class fusion positive B-ALL biologically and clinically phenocopies $\mathrm{Ph}^{+} \mathrm{ALL}$, one can speculate that early and continuous TKI administration in combination with chemotherapy may avoid HSCT in CR1 for a subset of ABL-class patients, allowing it to be reserved for patients at the highest risk of relapse.

With regards to the optimal chemotherapy backbone for pediatric $\mathrm{ABL}$-class patients, three regimens are currently being investigated in clinical trials: 1) the Total Therapy-based chemotherapy backbone from St. Jude Children's Research Hospital (clinicaltrials.gov identifer: NCT03117751); 2) the multinational European EsPhALL regimen as utilized in EsPhALL2010 (clinicaltrials.gov identifer: NCT00287105) and AALL1122 (clinicaltrials.gov identifer: NCT01460160); and 3) the COG AALL1131 modified augmented BFM backbone (clinicaltrials.gov identifer: NCT02883049). The latter two regimens are being com- pared in a randomized fashion in the phase III international trial for $\mathrm{Ph}^{+} \mathrm{ALL}$ in a non-inferior design (COG AALL1631; clinicaltrials.gov identifer: NCT03007147), which investigators plan to amend to also include ABLclass fusion patients. While awaiting the AALL1631 results to determine the optimal chemotherapy backbone for ABL-class patients, Cario et al. alluded to the high TRM rates when treating with the EsPhALL-inspired regimen, contributing to the poor outcomes of ABL-class patients. Similar findings have been observed in a recent publication from the AIEOP-BFM consortia; older adolescents aged 15-17 years also experienced significantly higher treatment-related deaths compared to their younger counterparts when treated on the AEIOP-BFM ALL 2000 chemotherapy backbone (without TKI), particularly in the HR arm that is the chemotherapy backbone to the EsPhALL regimen. ${ }^{17}$ Given that the prevalence of $\mathrm{Ph}$-like ALL rises with increasing age, toxicity remains a primary concern when adding TKI to the EsPhALL postinduction chemotherapy backbone for ABL-class patients. While therapy intensification has been an effective strategy to better outcomes in the past, in the modern era, we might have reached a plateau where further intensification is more likely to result in excessive toxicities rather than improve survival. Fortunately, the landscape of relapsed/refractory ALL therapy has witnessed major paradigm shifts with the emergence of immunotherapy. The bispecific CD3/CD19 T-cell engager, blinatumomab, or the anti-CD22 antibody drug conjugate, inotuzumab ozogamicin, in monotherapy or in combination with TKI, have been used in $\mathrm{Ph}^{+} / \mathrm{Ph}$-like ALL with promising early results. ${ }^{18-20}$ Therefore, incorporation of immunotherapy blocks intercalated within conventional chemotherapy backbone may represent an efficacious strategy to intensify therapy and reduce overlapping toxicities for ABL-class Ph-like ALL.

Cario et al. have provided an important dataset to fulfill the clinical portrait of the rare subset of ABL-class fusion positive B-ALL. The genomic landscape of Ph-like ALL and its associated poor prognosis have now been recognized for over a decade; thus, the time has come to act! The prospect of targeted therapy, immunotherapy and targeted use of CR1 HSCT, combined with lessons learned from previous $\mathrm{Ph}^{+}$ALL studies and international collaborations to conduct well-designed precision medicine trials, can establish pathways to increase cures for this high-risk ALL subset. One may dream that by improving outcomes of Ph-like ALL, we will be able to cure all ALL!

\section{References}

1. Hunger SP, Lu X, Devidas M, et al. Improved survival for children and adolescents with acute lymphoblastic leukemia between 1990 and 2005: a report from the children's oncology group. J Clin Oncol. 2012;30(14):1663-1669

2. Rheingold SR, Ji L, Xu X, et al. Prognostic factors for survival after relapsed acute lymphoblastic leukemia (ALL): A Children's Oncology Group (COG) study. J Clin Oncol. 2019;37(15_suppl): 1008-1008.

3. Den Boer ML, van Slegtenhorst M, De Menezes RX, et al. A subtype of childhood acute lymphoblastic leukaemia with poor treatment outcome: a genome-wide classification study. Lancet Oncol. 2009;10(2):125-134. 
4. Roberts KG, Li Y, Payne-Turner D, et al. Targetable kinase-activating lesions in Ph-like acute lymphoblastic leukemia. N Engl J Med. 2014;371(11):1005-1015

5. Reshmi SC, Harvey RC, Roberts KG, et al. Targetable kinase gene fusions in high-risk B-ALL: a study from the Children's Oncology Group. Blood. 2017;129(25):3352-3361.

6. Roberts KG, Gu Z, Payne-Turner D, et al. High Frequency and Poor Outcome of Philadelphia Chromosome-Like Acute Lymphoblastic Leukemia in Adults. J Clin Oncol. 2017;35(4):394-401.

7. Weston BW, Hayden MA, Roberts KG, et al. Tyrosine kinase inhibitor therapy induces remission in a patient with refractory EBF1-PDGFRB-positive acute lymphoblastic leukemia. J Clin Oncol. 2013;31(25):e413-416.

8. Tanasi I, Ba I, Sirvent N, et al. Efficacy of tyrosine kinase inhibitors in Ph-like acute lymphoblastic leukemia harboring ABL-class rearrangements. Blood. 2019;134(16):1351-1355

9. Cario G, Leoni V, Conter V, et al. Relapses and treatment-related events contributed equally to poor prognosis in children with ABLclass fusion positive B-cell acute lymphoblastic leukemia treated according to AIEOP-BFM protocols. Haematologica 2020;105(7): 1887-1894.

10. Schwab C, Ryan SL, Chilton L, et al. EBF1-PDGFRB fusion in pediatric B-cell precursor acute lymphoblastic leukemia (BCP-ALL): genetic profile and clinical implications. Blood. 2016;127(18):22142218.

11. Conter V, Bartram CR, Valsecchi MG, et al. Molecular response to treatment redefines all prognostic factors in children and adolescents with B-cell precursor acute lymphoblastic leukemia: results in 3184 patients of the AIEOP-BFM ALL 2000 study. Blood. 2010;115 (16):3206-3214.

12. Arico M, Valsecchi MG, Camitta B, et al. Outcome of treatment in children with Philadelphia chromosome-positive acute lymphoblastic leukemia. N Engl J Med. 2000;342(14):998-1006.

13. Schultz KR, Carroll A, Heerema NA, et al. Long-term follow-up of imatinib in pediatric Philadelphia chromosome-positive acute lymphoblastic leukemia: Children's Oncology Group study AALL0031.
Leukemia. 2014;28(7):1467-1471

14. Biondi A, Gandemer V, De Lorenzo P, et al. Imatinib treatment of paediatric Philadelphia chromosome-positive acute lymphoblastic leukaemia (EsPhALL2010): a prospective, intergroup, open-label, single-arm clinical trial. Lancet Haematol. 2018;5(12):e641-e652.

15. Slayton WB, Schultz KR, Kairalla JA, et al. Dasatinib Plus Intensive Chemotherapy in Children, Adolescents, and Young Adults With Philadelphia Chromosome-Positive Acute Lymphoblastic Leukemia: Results of Children's Oncology Group Trial AALL0622. J Clin Oncol. 2018;36(22):2306-2314

16. Roberts KG, Pei D, Campana D, et al. Outcomes of children with BCR-ABL1-like acute lymphoblastic leukemia treated with riskdirected therapy based on the levels of minimal residual disease. J Clin Oncol. 2014;32(27):3012-3020.

17. Testi AM, Attarbaschi A, Valsecchi MG, et al. Outcome of adolescent patients with acute lymphoblastic leukaemia aged 10-14 years as compared with those aged 15-17 years: Long-term results of 1094 patients of the AIEOP-BFM ALL 2000 study. Eur J Cancer. 2019;122:61-71.

18. Martinelli G, Boissel N, Chevallier P, et al. Complete Hematologic and Molecular Response in Adult Patients With Relapsed/Refractory Philadelphia Chromosome-Positive B-Precursor Acute Lymphoblastic Leukemia Following Treatment With Blinatumomab: Results From a Phase II, Single-Arm, Multicenter Study. J Clin Oncol. 2017;35(16):1795-1802.

19. Chiaretti S, Bassan R, Vitale A, et al. Dasatinib-Blinatumomab Combination for the Front-Line Treatment of Adult Ph+ ALL Patients. Updated Results of the Gimema LAL2116 D-Alba Trial. Blood. 2019;134(Suppl 1):740.

20. Jabbour E, Roberts KG, Sasaki K, et al. Inotuzumab Ozogamicin May Overcome the Impact of Philadelphia Chromosome-like Phenotype in Adult Patients with Relapsed/Refractory Acute Lymphoblastic Leukemia. Blood. 2019;134(Suppl_1):1641.

21. Roberts KG, Reshmi SC, Harvey RC, et al. Genomic and Outcome Analyses of Ph-like ALL in NCI Standard-risk Patients: A Report from the Children's Oncology Group. Blood. 2018;132:815-824.

\title{
NUP98 and KMT2A: usually the bride rather than the bridesmaid
}

\author{
Alexandre Fagnan ${ }^{1,2,3}$ and Thomas Mercher ${ }^{1,2,3.4}$ \\ ${ }^{1} I N S E R M$ U1170, Gustave Roussy Institute, Villejuif; ${ }^{2}$ Université Paris Diderot, Paris; ${ }^{3}$ Equipe labellisée Ligue Nationale Contre le \\ Cancer, Paris and ${ }^{4}$ Université Paris-Saclay, Villejuif, France
}

E-mail: THOMAS MERCHER - thomas.mercher@inserm.fr

doi:10.3324/haematol.2020.253476

$I^{n}$ n human hematopoietic malignancies, KMT2A and NUP98 are each independently targeted by numerous chromosomal alterations leading to the expression of fusion oncogenes. In this issue of Haematologica, Fisher and colleagues from J. Schwaller's team report the functional study and creation of an in vivo model ${ }^{1}$ for a unique fusion between these two genes ${ }^{2}$ showing that leukemia development by NUP98-KMT2A is not associated with classical KMT2A fusion mechanisms.

KMT2A (a.k.a. MLL) is a large protein of almost 4,000 amino acids that is processed by the endopeptidase Taspase1. It interacts with numerous proteins and assembles into large protein complexes (Figure 1). The functions of KMT2A include writing the H3K4me3 chromatin mark characteristic of active promoter regions through its Cterminal SET domain. In both lymphoid and myeloid malignancies, KMT2A is targeted by numerous chromosomal alterations resulting in the expression of fusion oncogenes with over 80 different partners in toto (https://mitelmandatabase.isb-cgc.org/). Experimental models have demonstrated that several fusions containing the $\mathrm{N}$-terminal portion of KMT2A and various partners [here termed KMT2A-X, where X is frequently AFF1, MLLT3, MLLT10 or MLLT1 in acute lymphoid leukemia patients, and MLLT3, MLLT10, MLLT1 or ELL in patients with acute myeloid leukemia (AML)] are important for disease development and maintenance. ${ }^{3,4}$

It has long been recognized that KMT2A-X fusions activate transcription of different $H O X$ genes (e.g. HOXC8, HOXA7, HOXA9, and HOXA10) and are associated with high expression of the HOX cofactor MEIS1. At the molecular level, at least two distinct mechanisms have been involved in KMT2A-X leukemogenic properties and the deregulated expression of KMT2A-X target genes (Figure 1). On the one hand, the first $145 \mathrm{~N}$-terminal amino acids of KMT2A interact with MEN1 and LEDGF to bind KMT2A target genes. ${ }^{5}$ On the other hand, most fusion partners of KMT2A belong to the transcription elongation machinery leading to the active recruitment of various factors including (i) the P-TEFb complex (comprising CDK9), which phosphorylates RNA polymerase II; and (ii) the histone methyltransferases DOT1L and NSD1, which catalyze H3K79me3 and H3K36me2 marks deposited in the body of actively transcribed genes. This 